\title{
Implementation and Evaluation of a Skills Training Program for Ultrasound-guided Vascular Access in Small Vessels Using a Low-Cost Cadaver Tissue Model
}

Michael Wagner ${ }^{1,2}$, Kirstin Hauser ${ }^{1}$, Francesco Cardona ${ }^{1}$, Georg M. Schmölzer²,3, Angelika Berger ${ }^{1}$, Monika Olischar ${ }^{1}$, Tobias Werther ${ }^{1}$

Division of Neonatology, Pediatric Intensive Care and Neuropediatrics, Department of Pediatrics, Medical University of Vienna, Austria; ${ }^{2}$ Centre for the Studies of Asphyxia and Resuscitation, Neonatal Research Unit, Royal Alexandra Hospital, Edmonton, AB, Canada

Department of Pediatrics, University of Alberta, Edmonton, AB, Canada

\section{BACKGROUND}

Central venous access in infants and children treated in neonatal and pediatric intensive care units is often require and difficult to obtain. Real-time ultrasound guidance for central venous catheterization (CVC) is considered one of the patient safety practices with strong evidence. Since the diameter of central veins in infants is much smaller than in adults, visualization and puncture of neonatal central veins require extensive training in this patient population. The purpose of this study was to assess the learning effect of a skills training program on ultrasound (US)-guided cannulation in a low-cost cadaver tissue model and an analysis of clinical utilization before and after the study period.

\section{METHODS}

In this prospective simulation-based study, we assessed the procedural competence of US-guided CVC in a low-cost cadaver tissue model using a before-after design. To assess the training program, we used a questionnaire and a performance checklist. Each participant attempted an US-guided puncture and insertion of a guide-wire in a small (two $\mathrm{mm}$ ) and a large (four $\mathrm{mm}$ ) tube using the in-plane and out-of-plane technique before and after a standardized teaching course.
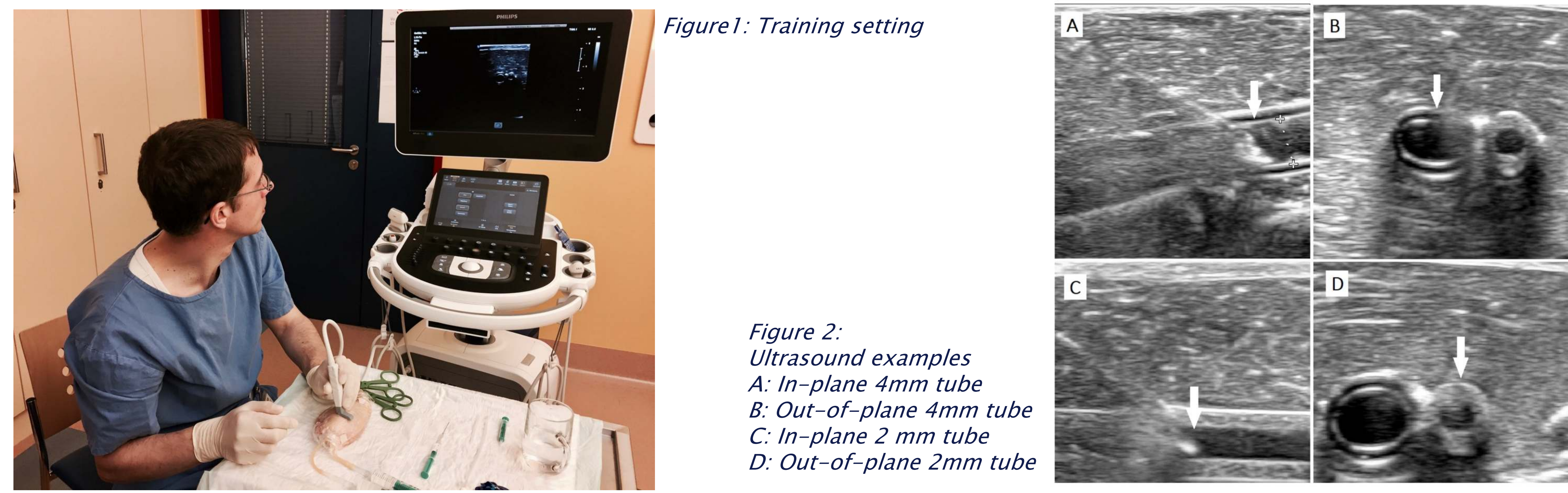

RESULTS

Thirty-nine staff physicians from the neonatal and pediatric intensive care units at the Medical University of Vienna participated in this study. There was a statistically significant reduction in failed attempts in the two $\mathrm{mm}$ vessels (in-plane: $\mathrm{p}=0.001$; out-of-plane: $\mathrm{p}=0.004$ ) after the initial training and teaching. No statistically significant difference was found in the four $\mathrm{mm}$ vessels (in-plane: $p=0.148$, out-of-plane: $p=0.069)$. The number of successful cannulations on the first attempt increased after teaching in all methods $(p=0.001)$. The number of US-guided CVC insertions at the neonatal care unit six months before $(n=8$ out of 28$)$ and after the training $(n=25$ out of 31$)$ significantly increased $(p<0.001)$.

\begin{tabular}{|c|c|c|c|c|c|c|c|c|c|c|c|c|}
\hline \multirow{2}{*}{ failed attempts, mean (range) } & \multicolumn{3}{|c|}{ in-plane $2 \mathrm{~mm}$} & \multicolumn{3}{|c|}{ in-plane $4 \mathrm{~mm}$} & \multicolumn{3}{|c|}{ out-of-plane $2 \mathrm{~mm}$} & \multicolumn{3}{|c|}{ out-of-plane $4 \mathrm{~mm}$} \\
\hline & pre & post & $p$-value & pre & post & $p$-value & pre & post & $\mathrm{p}$-value & pre & post & $\mathrm{p}$-value \\
\hline all participants & $2.1(0-3)$ & $1.1(0-3)$ & $0.001^{*}$ & $0.8(0-3)$ & $0.4(0-3)$ & 0.148 & $\begin{array}{c}1.8(0- \\
3)\end{array}$ & $1.2(0-3)$ & $0.004^{*}$ & $\begin{array}{c}0.6(0- \\
3)\end{array}$ & $0.3(0-3)$ & 0.069 \\
\hline \multicolumn{13}{|l|}{ subgroup (education) } \\
\hline resident $(n=22)$ & $2.0(0-3)$ & $1.0(0-3)$ & $0.026^{*}$ & $1.0(0-3)$ & $0.3(0-3)$ & $0.033^{*}$ & $\begin{array}{c}1.8(0- \\
3)\end{array}$ & $1.3(0-3)$ & 0.053 & $\begin{array}{c}0.4(0- \\
3)\end{array}$ & $0.3(0-3)$ & 0.52 \\
\hline fellow/attendant $(n=17)$ & $2.2(0-3)$ & $1.2(0-3)$ & $0.017^{*}$ & $0.6(0-3)$ & $0.5(0-3)$ & 0.893 & $\begin{array}{c}1.9(0- \\
3)\end{array}$ & $1.0(0-3)$ & $0.038^{*}$ & $\begin{array}{c}0.8(0- \\
3)\end{array}$ & $0.2(0-1)$ & 0.051 \\
\hline \multicolumn{13}{|l|}{ subgroup (US proficiency) } \\
\hline no $(n=9)$ & $1.8(0-3)$ & $0.8(0-3)$ & 0.150 & $0.6(0-3)$ & $0.3(0-2)$ & 0.843 & $\begin{array}{c}1.8(0- \\
3)\end{array}$ & $1.2(0-3)$ & 0.164 & $\begin{array}{c}1.0(0- \\
3)\end{array}$ & $0.3(0-2)$ & 0.085 \\
\hline yes $(n=30)$ & $2.2(0-3)$ & $1.2(0-3)$ & $0.003^{*}$ & $0.9(0-3)$ & $0.4(0-3)$ & 0.125 & $\begin{array}{c}1.9(0- \\
3)\end{array}$ & $1.1(0-3)$ & $0.014^{*}$ & $\begin{array}{c}0.4(0- \\
3)\end{array}$ & $0.3(0-3)$ & 0.256 \\
\hline \multicolumn{13}{|l|}{ subgroup (CVC experience) } \\
\hline none $(n=24)$ & $2.1(0-3)$ & $1.0(0-3)$ & $0.007^{*}$ & $0.9(0-3)$ & $0.5(0-3)$ & 0.167 & $\begin{array}{c}2.0(0- \\
3)\end{array}$ & $1.1(0-3)$ & $0.002^{*}$ & $\begin{array}{c}0.6(0- \\
3)\end{array}$ & $0.2(0-1)$ & $0.018^{*}$ \\
\hline yes $(n=15)$ & $2.1(1-3)$ & $1.1(0-3)$ & 0.054 & $0.8(0-3)$ & $0.3(0-3)$ & 0.554 & $\begin{array}{c}1.6(0- \\
3)\end{array}$ & $1.3(0-3)$ & 0.407 & $\begin{array}{c}0.5(0- \\
3)\end{array}$ & $0.5(0-3)$ & 1.0 \\
\hline
\end{tabular}

Table 1: Primary outcome $(* p<0.05$, US $=$ ultrasound, CVC $=$ central venous catheterization $)$

\section{CONCLUSION}

The implementation of a skills training for CVC in a cadaver tissue model was feasible and little time- and money-consuming. We were able to show a significantly decreased number of attempts until successful cannulation in the small vessel with an internal diameter of two $\mathrm{mm}$ after the standardized teaching session. Whereas total numbers of clinically CVC insertions were similar, we significantly increased the number of neonatologists (one before and four after the training) performing CVCs with the training. 